\title{
THE YOUNG PATIENT WITH CLAUDICATION
}

\author{
D T Reilly, John H N Wolfe
}

\section{Causes of intermittent claudication}

- Atherosclerosis

- Thromboembolism

- Buerger's disease

- Arteritis:

Systemic lupus erythematosus

Takayasu's disease

- Fibrosis:

Retroperitoneal

Radiation

- Developmental anomalies:

Coarctation

Persistent sciatic artery

Popliteal entrapment

- Trauma

- Cystic adventitial disease

All patients with intermittent claudication merit careful assessment, but the young claudicant patient in particular has much to gain from investigation, not only because an easily treatable lesion may be found but also because the long term outlook may be improved if risk factors are recognised and treated.

Most patients with intermittent claudication present at between 55 and 60 years of age, when the commonest cause is progression of atherosclerosis. In younger patients the other causes of claudication are proportionally more common. In this article we consider the patient who presents under the age of 50 .

A conservative approach is often appropriate for the older claudicant patient, particularly if clinical examination suggests a superficial femoral artery occlusion. Exercise, giving up smoking, and changes in the diet can be effective, and the natural history of intermittent claudication is that only about a quarter of patients continue to deteriorate. This conservative course is not appropriate for the young claudicant patient, in whom the symptoms more dramatically affect lifestyle, unusual diagnoses assume greater importance, and thorough investigation of the arterial system should be encouraged. Extensive non-invasive investigation is now possible and less invasive methods of treatment are more accepted.

\section{Diagnosis}

\section{Risk factors in the atherosclerotic} claudicant

- Smoking

- Diabetes mellitus

- Hypertension

- Hyperlipoproteinaemia

- Renal disease

- Family history of atherosclerosis
It is easy to overlook the symptoms in a 20 year old patient unless the possibility of intermittent claudication is kept in mind. There are, however, several possible causes of true intermittent claudication in a young person, including popliteal entrapment, cystic adventitial disease, and developmental anomalies. Venous claudication should not be overlooked in the differential diagnosis: there is the typical "bursting" sensation after walking that is relieved by raising the leg, and there is usually a history of injury or deep vein thrombosis. Atherosclerosis is common under the age of 50 , particularly among heavy smokers, but the diagnosis of atherosclerosis as the principal cause of symptoms in this age group should lead to a search for risk factors and associated disease; there may well be a history of angina or transient ischaemic attacks. Atherosclerotic claudication may later lead to stroke, myocardial infarction, and possibly loss of a limb. In younger patients atherosclerosis pursues a more aggressive course than in older ones, so every attempt should be made to improve prognosis. The overall five year mortality for patients who present with intermittent claudication is about $30 \%$.

\section{Examination and investigation}

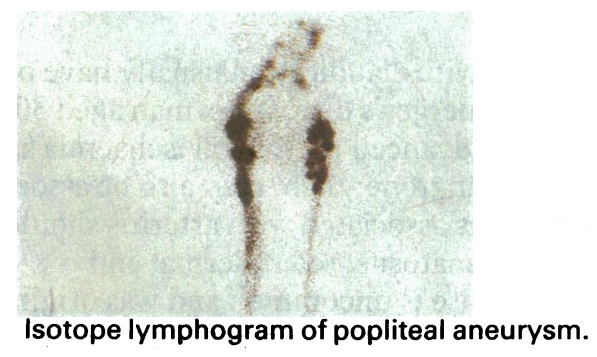

The clinical examination should pay particular attention to the presence of pulses, bruits, and signs of peripheral ischaemia. Aneurysms should be sought by careful palpation of the abdomen and the popliteal fossa; if a popliteal aneurysm is suspected it must be confirmed and treated because of the insidious embolisation of the distal run off vessels that occurs. By the time a popliteal aneurysm gives symptoms it may be too late for reconstruction.

The general examination should include careful cardiovascular evaluation, and orthopaedic examination may elicit or eliminate sciatica, spinal stenosis, and arthritis as causes of leg pain. 


\section{Specific conditions}

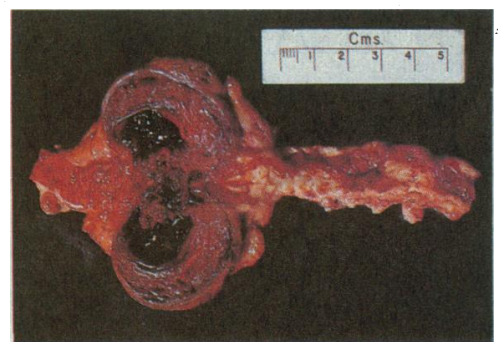

Histological specimen of thrombosed popliteal aneurysm.

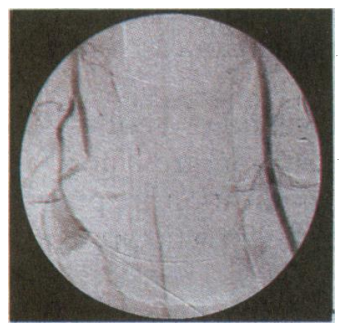

Digital subtraction arteriogram of popliteal entrapment.

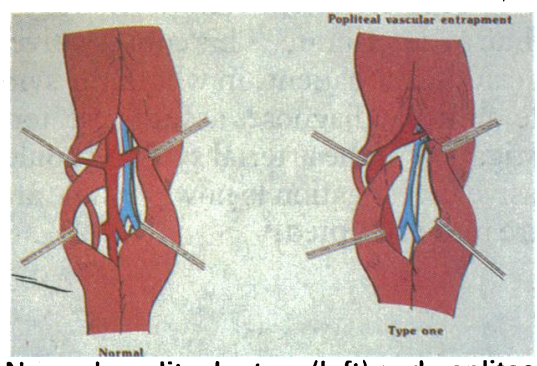

Normal popliteal artery (left) and popliteal entrapment (right).

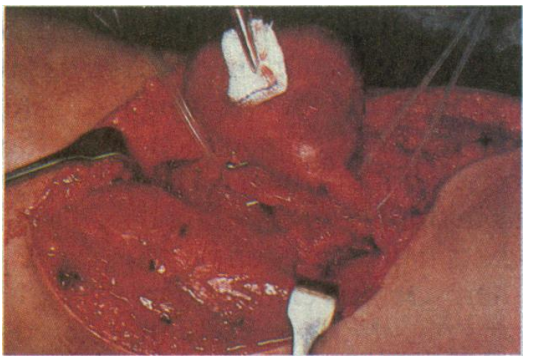

Operative photograph of popliteal aneurysm.

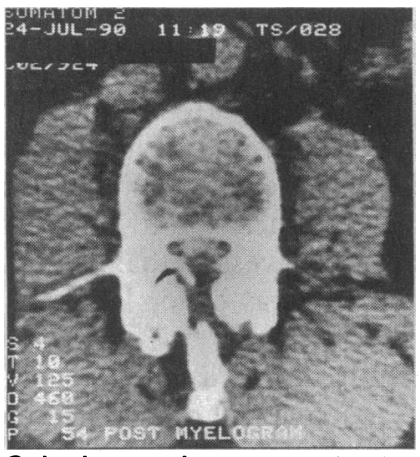

Spinal stenosis.

\section{Popliteal aneurysm}

The commonest cause of popliteal aneurysm is atherosclerosis, but other causes are popliteal artery entrapment, Marfan syndrome, and EhlersDanlos syndrome.

Atherosclerotic aneurysm - This is less common in the popliteal region than in the abdominal aorta, but $10 \%$ of patients with an abdominal aneurysm also have a popliteal aneurysm. Clinical suspicion is confirmed by ultrasound scanning, and arteriography should be carried out before operation, which entails ligation or resection of the aneurysm together with vein or synthetic bypass grafting.

Popliteal entrapment - The underlying lesion is an abnormal origin of the gastrocnemius, usually the medial head, which compresses the artery and causes the intermittent claudication. On clinical examination the pulses may be normal, but it may be possible to make the foot pulses disappear by holding the leg in full extension with plantar flexion or dorsiflexion of the foot. If left untreated the compressed artery may become occluded with thrombus or develop a poststenotic aneurysm. Arteriography of both legs should be done because the condition is often bilateral. At operation the aberrant muscle bundle is simply divided and-if the artery is occluded or aneurysmal - an end to end vein graft interposed.

Other causes of popliteal aneurysm - Patients with Marfan syndrome are usually tall, and have other typical features including arachnodactyly, high arched palate, and dislocated lenses, and as a rule do not present with claudication. This is also true of patients with the Ehlers-Danlos syndrome, who have hyperextensible joints and hyperelastic skin. Patients with mycotic aneurysms are unlikely to present with claudication, but may present with fever, malaise, and local pain.

\section{Other developmental anomalies}

Coarctation of the aorta may present in a young patient as bilateral intermittent claudication, but more usually presents as hypertension. Femoral pulses are absent or diminished, and collateral vessels may easily be found on the chest wall. A persistent sciatic artery is a rare cause of intermittent claudication in a young person, and can be diagnosed only by arteriography; on clinical examination the popliteal and foot pulses are absent.

\section{Cystic adventitial disease}

Cystic adventitial disease is an uncommon condition of unknown aetiology in which the lumen of the popliteal artery is compressed by a cyst (similar to a ganglion) developing in the tunica media. Onset of claudication is abrupt because of rupture of the cyst or haemorrhage within it. A characteristic curved indentation of the lumen is seen on arteriography (the "scimitar" sign). Duplex Doppler scanning also shows the cysts and is probably the best investigation. Percutaneous transluminal angioplasty is of no use because the lesion is outside the intima; the choice of treatment is either operation (with excision of the cystic area and vein grafting if necessary), or the more recently advocated percutaneous puncture and drainage under computed tomographic control.

\section{Trauma}

Most patients with vascular trauma present acutely and require urgent revascularisation; delayed presentation with vascular insufficiency may occur, however, after recovery from a major limb fracture that caused intimal damage. Even if the pulses are palpable, measurement of ankle pressures helps to confirm or exclude a serious problem if the patient has intermittent claudication. In a young person reconstructive surgery with an interposition graft is likely to be well worth while.

\section{Arteritis}

Patients with arteritis who present with claudication usually have other symptoms. The typical patient with Buerger's disease is a man aged 30 to 40 who smokes heavily and has signs of advanced peripheral ischaemia and sometimes early distal ulceration or gangrene; there may also be associated thrombophlebitis. Systemic conditions associated with arteritis should be sought, such as systemic lupus erythematosus, scleroderma, and rheumatoid arthritis. Takayasu's disease is uncommon and was originally described in young Oriental women. It also occurs in European races and is 


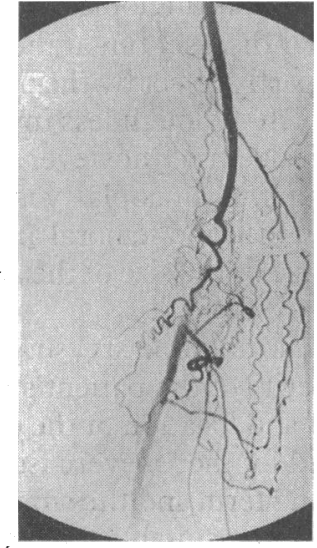

Right superficial femoral artery occlusion.

\section{Investigations}

\begin{tabular}{|l|}
\hline General investigations \\
- Full blood count \\
- Erythrocyte sedimentation rate \\
- Serum concentrations of: \\
Glucose \\
Lipoproteins \\
Triglycerides \\
Urea \\
Electrolytes \\
- Analysis of urine \\
- Chest radiography \\
- Electrocardiography \\
\hline
\end{tabular}

\section{Special investigations}

- Doppler ankle pressures

- Treadmill test

- Duplex Doppler ultrasound examination

- Arteriography:

Digital

Conventional

\section{Management}
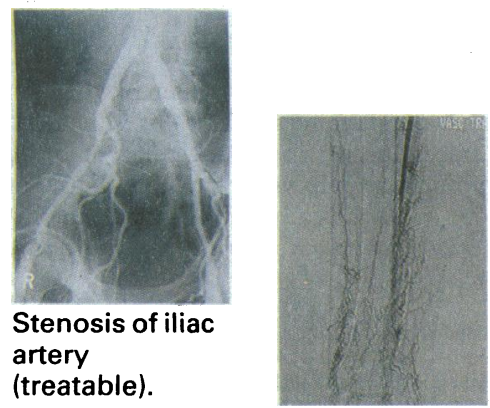

Buerger's disease (not treatable). an arteritis principally affecting the vessels of the aortic arch, but it can also cause occlusion of the aortoiliac segment. The main features are an unusual distribution of arterial stenoses or occlusions in a young patient and the finding of a high erythrocyte sedimentation rate on investigation. The arteritis is treated with steroids, and the patient must stop smoking. The 10 year survival for Takayasu's disease without operation is about $90 \%$.

\section{Other causes of vascular insufficiency}

Other causes of vascular insufficiency include such rarities as retroperitoneal fibrosis, which may be drug induced, idiopathic, or associated with granulomatous or malignant diseases. Aortoiliac stenosis can be caused by retroperitoneal fibrosis, in which case the clue will be diminished femoral pulses; standard bypass grafting should achieve a satisfactory result. Radiation fibrosis may also cause iliac occlusion, but is unlikely to be present in patients under 50 because of the long lag phase before radiation damage becomes apparent.

The most useful bedside test for the confirmation of a vascular problem is the measurement of ankle pressures by Doppler ultrasonography. Hand held Doppler flow detectors cost about $£ 300$ and should be available in all surgical clinics. If the ankle pressures are normal when the patient is at rest the measurements can be repeated after standardised exercise on a treadmill. If the ratio of ankle pressure to brachial pressure does not fall, a haemodynamically important arterial lesion may be excluded.

Further evaluation of vascular lesions is now less invasive than formerly in centres equipped with facilities for digital vascular imaging and duplex Doppler scanning; for the younger and otherwise fit claudicant patient an arteriogram is nevertheless indicated to establish a firm diagnosis and to see whether there is a superficial femoral artery lesion that is amenable to percutaneous transluminal angioplasty. A further indication for arteriography (rather than a "wait and see" policy) is if the patient has weak femoral pulses and symptoms of aortoiliac disease such as buttock or thigh claudication and erectile impotence. These patients do not usually improve with time, whereas with a superficial femoral artery occlusion the claudication distance may increase considerably as collateral vessels develop. Furthermore, percutaneous angioplasty and surgery are extremely successful in the aortoilial segment.

General investigation of the atherosclerotic patient should include an exercise stress electrocardiogram, and a chest radiograph is important because most of these patients are smokers. Routine blood tests should include a full blood count (to detect polycythaemia or thrombocythaemia) and measurement of the erythrocyte sedimentation rate (to confirm arteritis), of urea and electrolyte concentrations - together with analysis of the urine-(to detect occult renal disease, of the blood glucose concentration (to detect diabetes mellitus), and of fasting lipoprotein and triglyceride concentrations (to diagnose hyperlipoproteinaemia). If the history suggests embolisation, with a sudden onset of symptoms in a patient with an otherwise normal vascular tree, an echocardiogram is indicated to search for the source of the emboli. When no obvious cause for the occlusion in apparent primary thrombosis may have occurred as a result of antithrombin III, protein $\mathrm{C}$, or protein 5 deficiency. Homocystinuria may also predispose to thrombosis.

\section{General}

In the young atherosclerotic smoker the most important measures are to stop smoking, increase exercise, and change the diet. There is evidence that these are of long term benefit rather than punitive.

Though control of blood pressure and lipid concentrations may not be of such definite benefit to elderly people, there is increasing evidence that younger patients benefit from antihypertensive measures, dietary manipulation, and drugs to lower lipid concentrations such as cholestyramine (for hypercholesterolaemia), bezafibrate (for hypertriglyceridaemia), and gemfibrozil (for hyperlipidaemia).

\section{Specific measures in the atherosclerotic patient}

Though great claims are made for drugs to improve blood flow or tissue metabolism, none has so far been shown to produce a convincing and clinically useful effect. 
Patients with absent femoral pulses are readily treatable
The introduction of percutaneous transluminal angioplasty greatly improved the treatment of occlusive vascular disease, but at the cost of an increased number of arteriograms. This is partly offset by the increased use of intravenous digital vascular imaging, which - though less invasive - still requires a large dose of contrast medium. It does not, however, require admission to hospital. Percutaneous transluminal angioplasty is effective in treating short stenoses or occlusions of both iliac and femoral arteries, and requires an overnight stay in hospital because of the size of the arterial puncture.

If the lesion is not amenable to transluminal angioplasty, surgery has to be considered. This will depend on the severity of the patient's symptoms, his or her general condition, and the extent and location of the disease. Bypass grafting in the leg is usually reserved for more severe ischaemia because of the decrease in patency in the long term and the small but real risk of endangering the limb. A patient who is unsuitable for percutaneous transluminal angioplasty and is affected by only moderate claudication without severe ischaemia may have to come to terms with his or her disease.

Mr D T Reilly, FRCS, is consultant surgeon, Watford General Hospital, Watford, and Mr John H N Wolfe, FRCS, is consultant vascular surgeon, St Mary's Hospital, London.

The ABC of Vascular Diseases has been edited by Mr John H N Wolfe.

We acknowledge with thanks the assistance of the audiovisual department, St Mary's Hospital, London, in the preparation of the illustrations.

\section{ANY QUESTIONS}

Is a vaccine likely to be available to prevent women contracting toxoplasmosis during pregnancy?

The first attempt at vaccination with heat inactivated toxoplasma parasites was in 1924. Since then there have been many attempts at vaccination using live, attenuated, or killed toxoplasma or extracts of toxoplasma. Vaccination against toxoplasmosis makes sense in pregnant women as the problem is to avoid a primary infection. The problem is different in patients with AIDS, in whom disseminated toxoplasmosis often results from reactivation of previous infection. Thus a vaccine that may be useful to pregnant women may not help others at risk of serious toxoplasma infection.

It has been difficult to extend early experiments on toxoplasma vaccines. A vaccine using a mutant sensitive to temperature (ts-4) has been patented immunity, however, was not long lasting. Some killed vaccines were able to protect animals against lethal challenge with toxoplasma, but infection occurred and toxoplasma cysts were subsequently recovered from the animals' tissues. For pregnant women it is essential to prevent infection rather than clinical disease. Asymptomatic infection in the mother may result in a severely affected fetus. Therefore, despite its long history, toxoplasma vaccines have made little progress because the results of vaccination have not been good.

Manufacturers of vaccines will not be encouraged by such poor initia results. In addition, the public are more aware of injuries associated with vaccines, and thus vaccines need proper development. ${ }^{1}$ Nevertheless, some progress has been made in explaining some of these initial results. By using some of the newer molecular techniques a variety of individual antigens of toxoplasma have been identified. Some antigens are more protective and others are specific to a particular stage of toxoplasma infection..$^{2}$ Recently the 14 and the $35 \mathrm{kd}$ antigens have been found to be highly protective against toxoplasma infection. ${ }^{3}$

Indirect vaccination may also be useful in protecting pregnant women. If cats could be vaccinated so that they did not produce potentially infectious oocysts total toxoplasma infection would decline. Similarly, if farmers chose to vaccinate animals against toxoplasmosis the infection of humans would be less. For some animals there may be good economic reasons-for example, prevention of abortions - why vaccination may benefit the farmer.

The development of an appropriate vaccine for pregnant women depends on market forces. If there is sufficient demand from the public manufacturers will respond. A recent review of human vaccines that we need but do not have did not mention toxoplasma. ${ }^{2}$ Sadly, the future of a toxoplasma vaccine is more in the marketplace than in the laboratory. - D O HO-YEN, consultant microbiologist, Inverness
1 Robbins A. Progress towards vaccines we need and do not have. Lancet 1990;335:1436-8. 2 Hermentin $\mathrm{K}$, Aspock $\mathrm{H}$. Efforts towards a vaccine against Toxoplasma gondii: a review. Zentralbl Bakteriol Mikrobiol Hyg [A] 1988;269:423-36.

3 Araujo FG, Remington JS. Partially purified antigen preparations of Toxoplasma gondii protect against lethal infection in mice. Infect Immun 1984;45:122-6.

Does female sterilisation by occlusion of the fallopian tubes confer protection against pelvic inflammatory disease?

It is well established that in most cases pelvic sepsis is a result of ascending infection from the lower genital tract. The role of sexual activity in the genesis of infection is generally accepted. Sperm may provide a vector for transferring specific organisms, including Escherichia coli, Neisseria gonorrheae, and Ureaplasma urealyticum, to the uterine cavity and fallopian tubes. Infection due to Chlamydia trachomatis accounts for a high proportion of cases of pelvic inflammatory disease. The organism has been observed attached to sperm recovered from the peritoneal cavity at laparoscopy, and transmission of chlamydial infection by donor insemination has been described. Chlamydial infection is often asymptomatic but in as many as $10 \%$ of cases may lead to acute salpingitis. ${ }^{1}$

Barrier methods of contraception seem to be associated with a reduced risk of contracting pelvic inflammatory disease. This may be a reflection of the pattern of sexual behaviour of the individuals using such contraception rather than a specific protective effect of the method itself. Women who have been surgically sterilised also have a low incidence of pelvic inflammatory disease, but they probably belong to a group who are at low risk of contracting the disease in any case. Occlusion of the fallopian tubes cannot be expected to prevent the ascent of infective organisms in women who expose themselves to the risk of transmitted infection, and thus it is not surprising that there have been reports of acute pelvic infection in sterilised women. ${ }^{2}$ That sepsis may be found distal to the site of occlusion might be explained by the known potential for lymphatic spread of infection from the endometrial cavity. It is also theoretically possible that some interrupted tubes may contain a small fistula, allowing passage of organisms through the length of the fallopian tube to the peritoneal cavity beyond.

Currently available data suggest that about $8 \%$ of women in whom acute pelvic sepsis is diagnosed will have undergone sterilisation. Although this remains a safe method of contraception with a low incidence of infective complications, the possibility of acute salpingitis must be considered, despite a history of sterilisation, in all clinically symptomatic patients. MARK HAMILTON, senior lecturer and consultant gynaecologist, University of Aberdeen

1 Westrom L. Incidence, prevalence, and trends of acute pelvic inflammatory disease and its consequences in industrialised countries. Am F Obstet Gynecol 1980;138:880-92.

2 Vermesh M, Confino E, Boler LR, Friberg J, Gleicher N. Acute salpingitis in sterilised women. Obstet Gynecol 1987;69:265-7. 